médecine/sciences $1996 ; 12: 1035-6$

\title{
COURRIER
}

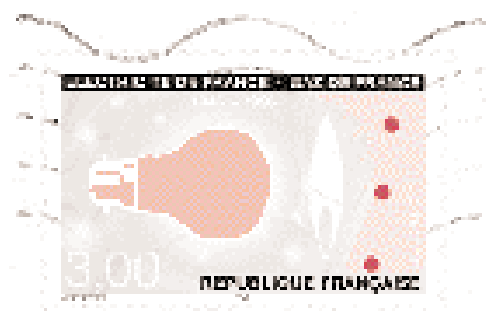

\section{Mésothéliome, amiante et cancer}

Dans son numéro d'avril 1996 médecine/sciences a consacré l'une de ses brèves au mésothéliome $\left(\mathrm{m} / \mathrm{s} n^{\circ} 4\right.$, vol. 12, p. 525), à l'occasion de la parution d'un article sur la mise en évidence de mutations du gène $N F 2$ dans ce type de tumeur [1]. La synthèse faite sur ce sujet reprend également des informations contenues dans un autre article sur «Amiante et mésothéliome", récemment publié par A. Knudson [2]. L'article de A. Knudson soulève quelques commentaires, plus particulièrement à propos des hypothèses sur les mécanismes de cancérogenèse de l'amiante.

Les mécanismes de toxicité des fibres d'amiante en général, et en cancérogenèse pleurale en particulier, ne sont pas aujourd'hui totalement élucidés. Certains auteurs considèrent que l'amiante joue un rôle épigénétique, agissant en tant que promoteur et favorisant la prolifération cellulaire, ce qui conduit à formuler l'hypothèse développée par Knudson, selon laquelle ces fibres n'agissent pas en tant que mutagène mais que les mutations résultent de l'accumulation de mutations «spontanées", au cours du temps. A l'appui de l'effet épigénétique, Knudson cite des résultats obtenus à partir d'un modèle assez complexe de cancérogenèse à deux étapes dans lequel l'amiante de type chrysotile agissait seulement en temps que co-cancérogène [3]. Dans cette étude, des explants de trachée ont été greffés, par implantation sous-cutanée, chez le rat syngénique. Des pastilles de cire contenant un hydrocarbure polyaromatique (diméthyl-benzanthracène) et des pastilles de gélatine chargée en fibres étaient insérées chirurgicalement, dans cet ordre, à un intervalle de 4 semaines. Les résultats ont montré que des carcinomes étaient observés chez les animaux recevant ces deux agents à des doses non tumorigènes à elles seules. Seules les tumeurs épithéliales ont été prises en considération; des sarcomes étant observés dans toutes les séries. D'autres éléments sont apportés à cette thèse: absence d'effet mutagène de l'amiante et stimulation de la prolifération cellulaire. En fait, les données de la littérature ne vont pas toutes dans ce sens et certains résultats montrent que l'amiante peut agir en tant que cancérogène complet, et qu'il peut aussi provoquer des mutations chromosomiques. Chez l'animal, des mésothéliomes sont observés après inoculation de doses aussi faibles que $50 \mu \mathrm{g}$ dans la cavité péritonéale; de même, sur cellules en culture, une transformation de cellules de mammifères est observée dans les systèmes classiques in vitro. Un certain nombre d'éléments expérimentaux permettent d'être plus critique sur le caractère uniquement promoteur de l'amiante. S'il est vrai qu'en présence d'amiante le taux de mutations géniques détectables sur des systèmes bactériens (test de Ames) ou de cellules eucaryotes (HGPRT, ouabaïne...) est rarement - mais pas toujours - supérieur au bruit de fond sans amiante (mutations spontanées), on ne peut nier que ces fibres soient potentiellement mutagènes. En effet, des mutations chromosomiques ont été mises en évidence à l'aide de modèles de cellules hybrides (délétions), ainsi qu'une perte d'hétérozygotie (locus HLA) dans des lymphocytes traités in vitro par des fibres. De plus, des anomalies chromosomiques, structurales et numériques, ainsi qu'une perturbation de la ségrégation des chromosomes au cours de la mitose ont été mises en évidence dans divers types cellulaires. Enfin, l'amiante provoque des dommages à l'ADN dans différents modèles cellulaires, dont les cellules mésothéliales. On peut, bien sûr, discuter des limites de ces modèles mais cela s'applique aussi aux modèles de "promotion". Dans l'état actuel de la connaissance, on doit rester prudent et prendre en considération tous ces effets, ce qui d'ailleurs n'exclut pas un effet épigénétique de l'amiante. Rien ne permet aujourd'hui d'éliminer une action au niveau du matériel génétique. Quant à l'effet des fibres sur la prolifération cellulaire, de nombreux travaux ont aussi montré un blocage de la prolifération cellulaire dont les mécanismes sont actuellement en cours d'étude.

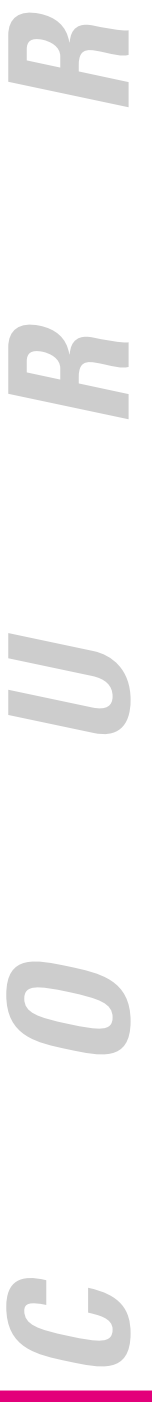


Concernant la connaissance actuelle de la nature des gènes modifiés dans le mésothéliome, je ne reviendrai pas sur ce qui a été dit sur le rôle potentiel de $N F 2$, confirmé par un autre travail dans lequel, de plus, de telles mutations n'étaient pas observées dans des cancers du poumon [6]. En revanche, les mutations sur le gène P53 ne semblent pas impliquées. Dans l'article mentionné par Knudson il y a certes des mutations détectées dans 2/4 lignées de mésothéliomes [7] mais une autre étude, portant sur un plus grand nombre de cas (17 individus), n'a pas mis en évidence de mutations spécifiques [8]. De même $W T-1$, s'il est muté dans un mésothéliome péritonéal n'est pas muté dans 32 mésothéliomes développés chez des sujets exposés à l'amiante [9].

Étant de longue date intéressée par la recherche portant sur cette thématique, j'ai beaucoup apprécié que $m e ́-$ decine/sciences aborde ce sujet, qui se trouve replacé aujourd'hui au cœur de débats sur le risque encouru par des expositions à de faibles concentrations en fibres minérales. Le récent article sur les mutations sur gène $N F 2$ est en fait le premier à mettre en évidence une modification spécifique dans le mésothéliome. Il ne permet toutefois pas de dire qu'il y a une prédisposition génétique à ce type de tumeur. On peut signaler à ce propos que récemment, Hirnoven et al. [10] ont observé que le risque était plus élevé chez des sujets qui présentaient un déficit combiné sur les gènes GSTM1 et NAT2 codant respectivement pour la glutathion Stransférase M1 et la N-acétyl transférase 2, mais les auteurs concluent que leurs résultats devraient être confirmés sur un plus grand nombre de cas

\section{RÉFÉRENCES}

1. Bianchi AB, Mitsunaga SI, Cheng JQ, et al. High frequency of inactivating mutations in the neurofibromatosis type 2 gene (NF2) in primary malignant mesothelioma. Proc Natl Acad Sci USA 1995; 92 : 10854-8.

2. Knudson A. Asbestos and mesothelioma: Genetic lessons from a tragedy. Proc Natl Acad Sci USA 1995; 92 : 10819-20.

3. Topping DC, Netteisheim P. Two-stage carcinogenesis studies with asbestos in Fischer 344 rats. J Natl Cancer Inst 1980; 65 : 627-30.

4. Jaurand MC. Particulate state carcinogenesis: a survey of recent studies on the mechanisms of action of fibres. IARC* Sci Pub 1989; 90 : 54-73.

* IARC: International Agency for Research on Cancer
5. Jaurand MC. Use of in vitro genotoxicity and cell transformation assays to evaluate potential carcinogenicity of fibres. IARC Sci Pub 1996 (sous presse).

6. Sekido Y, Pass HI, Bader S, Mew DJY, Christman MF, Gazdar AF, Minna JD. Neurofibromatosis type 2 (NF2) gene is somatically mutated in mesothelioma but not in lung cancer. Cancer Res 1995; 55 : 1227-31.

7. Cote RJ, Jhanwar SC, Novick S, Pellicer A. Genetic alterations of the p53 gene are a feature of malignant mesotheliomas. Cancer Res 1991; $51: 5410-6$.

8. Metcalf RA, Welsh JA, Bennett WP, et al. p53 and Kirsten-ras mutations in human mesothelioma cell lines. Cancer Res 1991; 52: 2610-5.

9. Park S, Schalling M, Bernard A, et al. The Wilms tumour gene WT1 is expressed in murine mesoderm-derived tissues and mutated in a human mesothelioma. Nature Genet 1993; 4: 415-20.

10. Hirvonen A, Pelin K, Tammilehto L, Karjalainen A, Mattson K, Linnainmaa K. Inherited GSTM1 and NAT2 defects as concurrent risk modifiers in asbestos-related human malignant mesothelioma. Cancer Res $1996 ; 95: 2981-3$.

\section{Marie-Claude Jaurand}

Directeur de recherche à l'Inserm, groupe d'étude sur la biologie de la cellule mésothéliale, Inserm U.139, faculté de médecine, 8, rue du Général-Sarrail, 94010 Créteil, France. 